\title{
Hamiltonian Analysis of Non-Linear Sigma Model on Supercoset Target
}

\author{
J. Kluson̆ * \\ Dipartimento di Fisica, \\ Universita' di Roma \& I. N. F. N Sezione di Roma 2, Tor Vergata \\ Via della Ricerca Scientifica, 100133 Roma ITALY \\ E-mail: Josef.Kluson@roma2.infn.it
}

\begin{abstract}
This paper is devoted to the study of the Hamiltonian formulation of non-linear sigma models on supercoset targets. We calculate the Poisson brackets of left-invariant currents. Then we introduce the Hamiltonian of the system and determine the equations of motion for left-invariant currents. We also determine the charge corresponding to the invariance of the action under global left multiplication.
\end{abstract}

KEYWORDS: string theory, non-linear sigma models.

\footnotetext{
${ }^{*}$ On leave from Masaryk University, Brno
} 


\section{Contents}

1. Introduction 1

2. Formulation of non-linear sigma model on supercoset targets 3

3. Hamiltonian formalism 6

4. Calculation of the current algebra 8

5. Hamiltonian and equations of motion 10

6. Global symmetry of the non-linear sigma model 12

\section{Introduction}

There has been recently great interest in non-linear sigma models on supercoset targets. These models have many applications in several branches of theoretical physics. For example, they can be used in the descriptions of the theory of quantum Hall efect [1, 2]. However the main motivation for the study of these models comes from string theory. In string theory the interest in the non-linear sigma models on supercoset targets is based on the remarkable observation that these models give description of the string theory in curved Ramond-Ramond backgrounds ${ }^{2}$. The Green-Schwarz action in $A d S_{5} \times S_{5}$ formulated by Metsaev and Tseytlin in [4] or its alternative form proposed and further developed in [5, 6, 7] takes the form of the sigma model on the coset superspace $P S U(2,2 \mid 4) / S O(1,4) \times S O(5)$. It was also soon discovered that the sigma model on simpler targets, the supergroups $P S L(n, n)$ has very rich and interesting structure [8, 9, 10]. In particular, it was shown that sigma models on cosets $G / H$ where $G$ is Ricci flat supergroup and $H$ is its bosonic subgroup are conformal to one loop - and it is expected to be conformal exactly given a suitable Wess-Zumino term. The important property of this construction is that the isotropy subgroup $H$ is fix point set of $Z_{4}$ grading of $G$.

It was further shown in [1] that the $Z_{4}$ grading is a key element for demonstration that the sigma model on $P S U(2,2 \mid 4) / S O(1,4) \times S O(5)$ is classically integrable

\footnotetext{
${ }^{2}$ For recent interestion discussion of properties of supercosets background, see [3].
} 
3 . This fact was also demonstrated in case of the pure spinor formulation of superstring on $A d S_{5} \times S_{5}$ [13, 14]. Moreover, since it was shown in [15, 16] that the pure spinor string on $A d S_{5} \times S_{5}$ is consistent quantum theory one can hope that the integrability persists in the quantum regime as well.

It was shown recently in two remarkable papers [17, 18 that the similar constructions hold for sigma models on spaces $G / H$ with more general $Z_{2 n}$ grading. For any $n$, the grading permits an introduction of certain preferred Wess-Zumino term. An existence of this term implies that the equations of motion can be put into the Lax form ensuring the integrability [18]. It was then shown in [17] that these models are conformally invariant up to one loop.

Due to these facts we mean that it is important to continue in the analysis of these sigma models. In particular, the knowledge of the classical Hamiltonian formalism of non-linear sigma models on the supercoset targets with $Z_{2 n}$ grading could be useful for further study of these models. For that reason the formulation of the the classical Hamiltonian analysis of the sigma model on supercoset targets is the main goal of this paper.

Let us outline the structure of the paper. In section (2) we introduce the sigma model action on supercoset targets following [17] and we briefly review its basic properties. Then in section (3) we formulate the Hamiltonian formalism of given theory. We introduce canonical variables using the method proposed in [19 ${ }^{4}$. In section (田) we calculate the Poisson bracket of the left-invariant currents. We check the validity of our calculations by comparing the Poisson bracket calculated here with the Poisson bracket derived in [22] and we find exact agreement. In section (5) we introduce the Hamiltonian for given system and also determine the equations of motion of the left-invariant currents. Finally, in section (6) we determine the charge corresponding to the invariance of the action under global left multiplication. We will calculate the Poisson brackets between these charges and spatial components of the left-invariant currents.

In summary, we mean that the properties of the non-linear sigma model on supercoset targets are very interesting and certainly deserve to be studied further. In particular, we hope that the results derived here could be helpful for an analysis of the quantum properties of these models. It would be also certainly very interesting to see whether the supercosets with $Z_{2 n}, n \neq 2$ grading correspond to some nontrivial background in string theory.

\footnotetext{
${ }^{3}$ The same conclusion was reached in 12 where the flat currents of the Green-Schwarz superstrings in $A d S_{5} \times S^{1}$ and $A d S_{3} \times S^{3}$ were constructed.

${ }^{4}$ For recent application of this method in string theory and quantum gravity, see [20, 21, 22, 23, 24.
} 


\section{Formulation of non-linear sigma model on supercoset tar- gets}

We begin this section with the brief review of properties of supercosets. We do not want to give the complete outline of this subject. We rather focus on properties of the supercoset that are necessary for definition of the non-linear sigma model on it.

Let us consider an associative Grassmann algebra $\Lambda=\Lambda_{0}+\Lambda_{1}$, where $\Lambda_{0}$ (resp. $\Lambda_{1}$ ) consists of commuting (resp. anticommuting) elements. Given a supermatrix $X=\left(\begin{array}{ll}A & B \\ C & D\end{array}\right)$ that belongs to the supergroup $G$ we define it to be even (odd) if $A, D \in \Lambda_{0}\left(\Lambda_{1}\right)$ and $B, C \in \Lambda_{1}\left(\Lambda_{0}\right)$. We introduce the notation $|X| \equiv \operatorname{deg}(X)$ and we write $|X|=0$ if $X$ is even matrix and $|X|=1$ if it is odd matrix. Then we can define the supertrace as

$$
\operatorname{Str}(X)=\operatorname{Tr}(A)-(-1)^{|X|} \operatorname{Tr} D
$$

This supertrace satisfies an important property

$$
\operatorname{Str}(X Y)=(-1)^{|X||Y|} \operatorname{Str}(Y X)
$$

The relation between a supergroup and superalgebra is similar to the bosonic case. The supergroup $G$ associated to the superalgebra $\mathbf{g}$ is the exponential mapping of the even subsuperalgebra of Grassmann envelope $\Lambda \otimes \mathbf{g}$

$$
g=\exp \left(x^{A} T_{A}\right)
$$

where $T_{A}$ generate the Lie superalgebra $\mathbf{g}=\mathbf{g}^{\text {even }}+\mathbf{g}^{\text {odd }}$ corresponding to $G$. We denote $|A|$ the grade of $T_{A}$ in the Lie superalgebra. Then $T_{A}$ satisfy the supercommutation relationship

$$
\left[T_{A}, T_{B}\right]=T_{A} T_{B}-(-1)^{|A||B|} T_{B} T_{A}=f_{A B}^{C} T_{C},
$$

where the structure constraints $f_{A B}^{C}$ satisfy the graded Jacobi identity

$$
0=(-1)^{|A||C|} f_{A D}^{E} f_{B C}^{D}+(-1)^{|B||A|} f_{B D}^{E} f_{C A}^{D}+(-1)^{|C||B|} f_{C D}^{E} f_{A B}^{D}
$$

The $x^{A}$ commute (resp. anticommute) whenever $T_{A}$ are graded even (resp. odd).

As the next step let us now presume that $\mathbf{g}$ is $Z_{2 n}$ graded and $\mathbf{h}$, the complexified Lie algebra of the isotropy subgroup $H$ is the subspace of grade zero ${ }^{5}$. That is we suppose that $\mathbf{g}$ may be written as a direct sum

$$
\mathbf{g}=\mathbf{g}_{0}+\mathbf{g}_{1}+\ldots+\mathbf{g}_{2 n-1}
$$

\footnotetext{
${ }^{5}$ The $Z_{2 n}$ grading is defined by automorphism $\Omega: \mathbf{g} \rightarrow \mathbf{g}$ such that $[\Omega(X), \Omega(Y)]=$ $\Omega([X, Y]), \Omega^{2 n}=1$ and $\Omega^{k} \neq 1$ for all $k<2 n$.
} 
of vector subspaces where $\mathbf{g}_{0}=\mathbf{h}$ and that this decomposition respects the graded Lie bracket

$$
\left[\mathbf{g}_{r}, \mathbf{g}_{s}\right] \subset \mathbf{g}_{r+s \bmod 2 n} .
$$

We presume that this grading is compatible with the splitting to Grassmann odd and even variables, namely

$$
\mathbf{g}_{2 s} \subset \mathbf{g}^{\text {even }}, \mathbf{g}_{2 s+1} \subset \mathbf{g}^{\text {odd }}, \text { for } \mathrm{s}=0,1, \ldots, n-1 \text {. }
$$

The basis $T_{A}$ can be chosen to be a disjoint union of bases of $\mathbf{g}_{r}$. The basis element of $\mathbf{g}_{r}$ will be denoted as $T_{i_{r}}$ where $i_{r}=1 \ldots$ dim $\mathbf{g}_{r}$. We also use following conventions for the naming of indices:

$$
\begin{array}{r}
i_{A}, j_{B}, \ldots \text { generators of } \mathbf{g}, \\
i_{0} \equiv x, j_{0} \equiv y, \ldots \text { generators of } \mathbf{h} \\
i_{r}, j_{r}, \ldots \text { generators of } \mathbf{g}_{r}, \\
r=1, \ldots, 2 n-1 .
\end{array}
$$

We also presume that the supertrace respect the grading in a sense ${ }^{6}$

$$
\operatorname{Str} X Y=\operatorname{Str} \Omega(X) \Omega(Y) .
$$

Let us now presume that $X \in \mathbf{g}_{r}, Y \in \mathbf{g}_{s}$ so that

$$
\Omega(X)=e^{\frac{i r \pi}{n}} X, \Omega(Y)=e^{\frac{i s \pi}{n}} X .
$$

Then using (2.10) we immediately obtain

$$
\operatorname{Str} X Y=0 \text { unless } \mathrm{r}+\mathrm{s}=0 \bmod 2 \mathrm{n} .
$$

Now we are ready to formulate the sigma model on $G / H$, following [17]. We express the sigma model on $G / H$ in terms of a dynamical field $g\left(x^{\mu}\right) \in G$ where $x^{\mu}, \mu=0,1$ are worldsheet coordinates. We write

$$
J_{\mu}=g^{-1} \partial_{\mu} g \in \mathbf{g}
$$

Note that this current is invariant under the global left action

$$
g^{\prime}=U g, U \in G \text {. }
$$

We can decompose the current into currents of defined grade

$$
J_{\mu}=J_{\mu}^{(0)}+\tilde{J}_{\mu}, J_{\mu}^{(0)} \in \mathbf{h}, \tilde{J}_{\mu} \in \mathbf{g} / \mathbf{h},
$$

\footnotetext{
${ }^{6}$ This can be easily demonstrated in case when the action of $\Omega$ on $X$ can be represented as an operation of conjugation $\Omega(X)=M X M^{-1}$ for some matrix $M$.
} 
where

$$
J_{\mu}^{(0)}=J_{\mu}^{x} T_{x}, \tilde{J}_{\mu}=\sum_{r=1}^{2 n-1} \sum_{i_{r}=1}^{\operatorname{dim} \mathbf{g}_{r}} J_{\mu}^{i_{r}} T_{i_{r}} \equiv \sum_{r=1}^{2 n-1} J_{\mu}^{(r)} .
$$

In what follows we use the Einstein summation convention. Explicitly, we define

$$
\begin{aligned}
& \sum_{r=1}^{2 n-1} \sum_{i_{r}=1}^{\operatorname{dim} \mathbf{g}_{r}} J_{\mu}^{i_{r}} T_{i_{r}} \equiv \sum_{r} J_{\mu}^{i_{r}} T_{i_{r}}, \\
& \sum_{A=0}^{2 n-1} \sum_{i_{A}=1}^{\operatorname{dim} \mathbf{g}_{A}} J_{\mu}^{i_{A}} T_{i_{A}} \equiv \sum_{A} J_{\mu}^{i_{A}} T_{i_{A}}, i_{0} \equiv x .
\end{aligned}
$$

Let us now study the transformation properties of the current under the local right action $g^{\prime}=g h, h(x) \in H$. Using the definition of $J$ we easily obtain

$$
J_{\mu}^{\prime}=h^{-1} J_{\mu} h+h^{-1} \partial_{\mu} h .
$$

Then using the fact that $\Omega(h)=h$ it is easy to determine the rules how currents $J^{(0)}$ and $\tilde{J}$ transform under local right action

$$
\begin{aligned}
J_{\mu}^{(0)} & =h^{-1} \partial_{\mu} h+h^{-1} J_{\mu}^{(0)} h, \\
\tilde{J}_{\mu}^{\prime} & =h^{-1} \tilde{J}_{\mu} h .
\end{aligned}
$$

The next important object that is needed for definition of the non-linear sigma model on supercoset target is the metric

$$
K_{i_{A} j_{B}}=\operatorname{Str}\left(T_{i_{A}} T_{j_{B}}\right) .
$$

This metric has the nonzero components

$$
K_{i_{r} j_{2 n-r}}=(-1)^{|r|} K_{j_{2 n-r} i_{r}}, K_{x y}=K_{y x}
$$

as follows from (2.10). Using (2.21) we define two form field $B$ as

$$
B_{i_{r} j_{2 n-r}}=q_{r} K_{i_{r} j_{2 n-r}}, q_{2 n-r}=-q_{r} .
$$

Note also that (2.22) obeys the graded antisymmetry property

$$
B_{i_{r} j_{2 n-r}}=-(-1)^{|r|} B_{j_{2 n-r} i_{r}}
$$

that follows from (2.21) and from the fact that $q_{2 n-r}=-q_{r}$. Then we can write the action for non-linear sigma model on supercoset target in the form

$$
S=-\int d^{2} x \sum_{r}\left(\frac{1}{2} \eta^{\mu \nu} K_{i_{r} j_{2 n-r}} J_{\mu}^{i_{r}} J_{\nu}^{j_{2 n-r}}+\frac{1}{2} \epsilon^{\mu \nu} B_{i_{r} j_{2 n-r}} J_{\mu}^{i_{r}} J_{\nu}^{j_{2 n-r}}\right) .
$$

Equivalently, using (2.20) and (2.21) the action (2.24) can be written as

$$
S=-\int d^{2} x \sum_{r} \operatorname{Str}\left(\frac{1}{2} \eta^{\mu \nu} J_{\mu}^{(r)} J_{\nu}^{(2 n-r)}+\frac{1}{2} \epsilon^{\mu \nu} q_{r} J_{\mu}^{(r)} J_{\nu}^{(2 n-r)}\right) .
$$




\section{Hamiltonian formalism}

Next step is to determine the canonical variables and define corresponding conjugate momenta. To do this we follow the approach introduced in [19]. We start with the fact that the current $J_{\mu}$ is flat

$$
\partial_{\mu} J_{\nu}-\partial_{\nu} J_{\mu}+\left[J_{\mu}, J_{\nu}\right]=0 .
$$

With the help of this identity we can express $J_{0}$ as a function of $J_{1}$ if we define the operator $D$ as

$$
\partial_{0} J_{1}=\partial_{1} J_{0}+\left[J_{1}, J_{0}\right] \equiv D J_{0} .
$$

Now we presume that the currents $J_{\mu}$ obey appropriate boundary conditions so that we can introduce the inverse operator $D^{-1}$ to express $J_{0}$ as

$$
J_{0}=D^{-1}\left(\partial_{0} J_{1}\right) .
$$

If we insert (3.3) into the action (2.25) we obtain the action that contain the dynamical variable $J_{1}$ and its time derivative $\partial_{0} J_{1}$. Then using the standard method we can define the momenta conjugate to $J_{1}$. More precisely, let us extract the part of the action (2.25) that contains the time components of the currents

$$
\begin{array}{r}
S_{0}=\frac{1}{2} \int d^{2} x \sum_{r} \operatorname{Str}\left(J_{0}^{(r)} J_{0}^{(2 n-r)}-g_{r} J_{0}^{(r)} J_{1}^{(2 n-r)}\right)=\frac{1}{2} \int d^{2} x\left(\tilde{J}_{0} \tilde{J}_{0}+\tilde{J}_{0} \hat{J}_{1}\right)= \\
=\frac{1}{2} \int d^{2} x\left[D^{-1}\left(\tilde{\partial}_{0} J_{1}\right) D^{-1}\left(\partial_{0} J_{1}\right)+D^{-1}\left(\partial_{0} J_{1}\right) \hat{J}_{1}\right],
\end{array}
$$

where in the second step we have used (2.10) and where we have also defined

$$
\hat{J}_{1}=\sum_{r} q_{2 n-r} J_{1}^{2 n-r} .
$$

Finally the formula $D^{-1}\left(\partial_{0} J_{1}\right)$ means that we project to the superalgebra of the coset $\mathrm{g} / \mathbf{h}$. Then it is simple task to define the momentum $\Pi_{J}$ as a variation of the action (3.4) with respect to $\partial_{0} J_{1}$ and we get

$$
\Pi_{J}=\frac{\delta S}{\delta \partial_{0} J_{1}}=-D^{-1}\left(D^{-1}\left(\tilde{\partial}_{0} J_{1}\right)+\hat{J}_{1}\right),
$$

where we have used the fact that

$$
\int d^{2} x \operatorname{Str}\left(D^{-1}(\delta X)(\ldots)\right)=-\int d^{2} x \operatorname{Str}\left(\delta X D^{-1}(\ldots)\right) .
$$

If we now act with $D$ on (3.6) from the left we obtain

$$
\left.D \Pi_{J}=-D^{-1} \tilde{\partial}_{0} J_{1}\right)-\hat{J}_{1}
$$


that allows us to express $\tilde{J}_{0}$ as function of canonical variables $\Pi_{J}$ and $J_{1}$

$$
\tilde{J}_{0}=-D \Pi_{J}-\hat{J}_{1}
$$

To proceed further we expand $J_{\mu}$ and $\Pi_{J}$ as

$$
\Pi_{J}=\sum_{A} \Pi^{i_{A}} T_{i_{A}}, \tilde{J}_{0}=\sum_{r} J_{0}^{i_{r}} T_{i_{r}}, J_{1}=\sum_{A} J_{1}^{i_{A}} T_{i_{A}}, \hat{J}_{1}=\sum_{r} q_{r} J^{i_{r}} T_{i_{r}} .
$$

Then $D \Pi_{J}$ takes the form

$$
\begin{array}{r}
D \Pi_{J}=\partial_{1} \Pi_{J}+\left[J_{1}, \Pi_{J}\right]= \\
\sum_{A}\left(\partial_{1} \Pi^{i_{A}} T_{i_{A}}+\sum_{B} J_{1}^{j_{B}} \Pi^{k_{A-B}} f_{j_{B} k_{A-B}}^{i_{A}} T_{i_{A}}\right)
\end{array}
$$

using the fact that the structure constants have the form $f_{i_{A} j_{B}}^{k_{A+B}}$ as follows from (2.7). Then with the help of (3.11) and (3.10) the equation (3.9) is equal to

$$
\begin{aligned}
-J_{0}^{i_{r}} & =\partial_{1} \Pi^{i_{r}}+\sum_{A} J_{1}^{j_{A}} \Pi^{k_{r-A}} f_{j_{A} k_{r-A}}^{i_{r}}+q_{r} J_{1}^{i_{r}}, \\
\Phi^{x} & =\partial_{1} \Pi^{x}+\sum_{A} J_{1}^{i_{A}} \Pi^{j_{2 n-A}} f_{i_{A} j_{2 n-A}}^{x} \approx 0,
\end{aligned}
$$

where the absence of $J_{0}^{x}$ in the action implies an existence of the primary constraint $\Phi^{x}$.

Let us now introduce the equal-time graded Poisson bracket that for two classical observables $F, G$ depending on the phase super-space variables $J_{1}^{i_{A}}, \Pi_{j_{A}}$ is defined as

$$
\{F, G\}=(-1)^{|F||A|} \sum_{A}\left[\frac{\partial^{L} F}{\partial J_{1}^{i_{A}}} \frac{\partial^{L} G}{\partial \prod_{i_{A}}}-(-1)^{|A|} \frac{\partial^{L} F}{\partial \prod_{i_{A}}} \frac{\partial^{L} G}{\partial J_{1}^{i_{A}}}\right]
$$

where the superscript $L$ denotes left derivation. For the components $J_{1}=\sum_{A} J_{1}^{i_{A}} T_{i_{A}}$, $\Pi_{J}=\sum_{A} \Pi^{i_{A}} T_{i_{A}}=\sum_{A} K^{i_{A} j_{2 n-A}} \prod_{j_{2 n-A}} T_{i_{A}}$, the above PB's read

$$
\left\{J_{1}^{i_{A}}(x), \Pi_{j_{B}}(y)\right\}=(-1)^{|A|} \delta_{B}^{A} \delta_{j}^{i} \delta(x-y),
$$

where $\Pi_{i_{A}}$ is defined as

$$
\Pi_{i_{A}}=K_{i_{A} j_{2 n-A}} \Pi^{j_{2 n-A}} .
$$

We again stress that $A$ that labels the graded subspaces $\mathbf{g}^{(A)}$ is in the one to one correspondence with Grassmann property of given elements, namely $A$ odd labels Grassmann odd subalgebra and $A$ even labels the Grassmann even subalgebra.

Let us now define

$$
J_{i_{t}}^{\mu} \equiv K_{i_{r} j_{2 n-r}} J_{\mu}^{j_{2 n-r}}
$$


Then we can rewrite (3.12) in an alternative form

$$
\begin{aligned}
& J_{i_{r}}^{0}=-\partial_{1} \Pi_{i_{r}}-\sum_{A} J_{1}^{k_{A}} f_{i_{r} k_{A}}^{j_{r+A}} \Pi_{j_{r+A}}+q_{r} J_{i_{r}}^{1}, \\
& \Phi_{x}=\partial_{1} \Pi_{x}+\sum_{A} J_{1}^{k_{A}} f_{x k_{A}}^{j_{A}} \Pi_{j_{A}}
\end{aligned}
$$

using the fact that $q_{r}=-q_{2 n-r}$.

\section{Calculation of the current algebra}

In this section we will calculate the Poisson brackets between currents $J_{\mu}^{i_{A}}$ using the canonical Poisson brackets derived in the previous section. We start with the Poisson bracket of the form $\left\{J_{i_{r}}^{0}(x), J_{1}^{j_{s}}(y)\right\}$. These Poisson brackets can be easily calculated with the help of (3.14) and (3.17) and we obtain

$$
\begin{aligned}
& \left\{J_{i_{t}}^{0}(x), J_{1}^{j_{t}}(y)\right\}=\delta_{i}^{j} \partial_{x} \delta(x-y)+f_{i_{t}}^{j_{t}} J_{1}^{x}(x) \delta(x-y), \\
& \left\{J_{i_{t}}^{0}(x), J_{1}^{j_{r}}(y)\right\}=f_{i_{t} l_{r-t}}^{j_{r}} J_{1}^{l_{r-t}}(x) \delta(x-y), \text { for } \mathrm{r} \neq \mathrm{t}, \\
& \left\{J_{i_{t}}^{0}(x), J_{1}^{x}(y)\right\}=f_{i_{t} j_{2 n-t}}^{x} J_{1}^{j_{2 n-t}}(x) \delta(x-y) .
\end{aligned}
$$

Using (3.16) we obtain the alternative form of Poisson bracket (4.1)

$$
\begin{aligned}
\left\{J_{0}^{i_{2 n-t}}(x), J^{j_{t}}(y)\right\} & =K^{i_{2 n-t} j_{t}} \partial_{x} \delta(x-y)+J_{1}^{x}(x) f_{x k_{2 n-t}}^{i_{2 n-t}} K^{k_{2 n-t} j_{t}}, \\
\left\{J_{0}^{i_{t}}(x), J_{1}^{j_{r}}(y)\right\} & =J_{1}^{l_{r+t}}(x) f_{l_{r+t} k_{2 n-r}}^{i_{t}} K^{k_{2 n-r} j_{r}}, \\
\left\{J_{0}^{i_{t}}(x), J_{1}^{x}(y)\right\} & =J_{1}^{j_{t}}(x) f_{j_{t} y}^{i_{t}} K^{y x} \delta(x-y),
\end{aligned}
$$

where we have also used the fact that $2 n+t \sim t$.

In the same way we can determine the Poisson bracket between $\Phi_{x}$ and $J_{1}^{i_{r}}, J_{1}^{y}$

$$
\begin{aligned}
\left\{\Phi_{z}(x), J_{1}^{v}(y)\right\} & =-\delta_{z}^{v} \partial_{x} \delta(x-y)-f_{z w}^{v} J_{1}^{w}(x) \delta(x-y), \\
\left\{\Phi_{x}(x), J_{1}^{i_{r}}(y)\right\} & =-f_{x j_{r}}^{i_{r}} J_{1}^{j_{r}}(x) \delta(x-y) .
\end{aligned}
$$

These Poisson brackets demonstrate how currents $J_{1}^{i_{r}}$ transform under the gauge transformations generated by $\Phi_{x}$. It is also clear that $J_{0}^{i_{r}}$ has to transform in the same way so that

$$
\left\{\Phi_{x}(x), J_{0}^{i_{r}}(y)\right\}=-f_{x j_{r}}^{i_{r}} J_{0}^{j_{r}}(x) \delta(x-y)
$$

or equivalently

$$
\left\{\Phi_{x}(x), J_{i_{r}}^{0}(y)\right\}=J_{j_{r}}^{0}(x) f_{x i_{r}}^{j_{r}} \delta(x-y)
$$


Now we will calculate the Poisson brackets between zero components of the currents $J_{0}^{i_{A}}$. Let us start with the Poisson bracket $\left\{J_{i_{t}}^{0}(x), J_{j_{2 n-t}}^{0}(y)\right\}$. After straightforward, but slightly involved calculation we obtain

$$
\left\{J_{i_{t}}^{0}(x), J_{j_{2 n-t}}^{0}(y)\right\}=(-1)^{|t|} \Phi_{x}(x) f_{i_{t} j_{2 n-t}}^{x} \delta(x-y),
$$

where we have used (3.14) and (3.17) and also the fact that the structure functions of the supercoset obey the relation

$$
f_{i_{A} j_{B}}^{k_{A+B}} K_{k_{A+B} l_{C}}=-(-1)^{|B||C|} f_{i_{A} l_{C}}^{k_{A+C}} K_{k_{A+C} j_{B}}
$$

and the graded Jacobi identity

$$
0=(-1)^{|A||C|} f_{i_{A} j_{B+C}}^{m_{A+B+C}} f_{k_{B} l_{C}}^{j_{B+C}}+(-1)^{|B||A|} f_{k_{B} j_{A+C}}^{m_{A+B+C}} f_{l_{C} i_{A}}^{j_{A+C}}+(-1)^{|C||B|} f_{l_{C} j_{A+B}}^{m_{A+B+C}} f_{i_{A} k_{B}}^{j_{A+B}},
$$

where we have also used (2.7). Finally note that (4.6) can be written in the form

$$
\left\{J_{0}^{i_{2 n-t}}(x), J_{0}^{j_{t}}(y)\right\}=-\Phi^{x}(x) f_{x k_{2 n-t}}^{i_{2 n-t}} K^{k_{2 n-t} i_{t}},
$$

where $\Phi^{x}=\Phi_{y} K^{y x}$.

As the next step we will calculate the Poisson bracket

$$
\left\{J_{i_{t}}^{0}(x), J_{j_{s}}^{0}(y)\right\}, t \neq s .
$$

This Poisson bracket can be calculated exactly in the same way as in the previous cases however now the result strongly depends on the value of $q_{t}$ that appears in (2.22) and that according to [17, 18] is equal to

$$
q_{s}=1-\frac{s}{n} \text {. }
$$

More precisely it turns out that for $t+s<2 n$ the Poisson bracket (4.10) takes the form

$$
\left\{J_{i_{t}}^{0}(x), J_{j_{s}}^{0}(y)\right\}=-(-1)^{|s||t|}\left(J_{l_{t+s}}^{1}+J_{l_{t+s}}^{0}\right)(x) f_{i_{t} j_{s}}^{l_{t+s}} \delta(x-y)
$$

while for $t+s>2 n$ it is equal to

$$
\left\{J_{i_{t}}^{0}(x), J_{j_{s}}^{0}(y)\right\}=(-1)^{|s||t|}\left(J_{l_{t+s}}^{1}-J_{l_{t+s}}^{0}\right)(x) f_{i_{t} j_{s}}^{l_{t+s}} \delta(x-y) .
$$

It will be also useful to express (4.12) and (4.13) in the alternative form as

$$
\begin{aligned}
& \left\{J_{0}^{i_{t}}(x), J_{0}^{j_{s}}(y)\right\}=\left(J_{0}^{l_{t+s}}+J_{1}^{l_{t+s}}\right)(x) f_{l_{t+s} k_{2 n-s}}^{i_{t}} K^{k_{2 n-s} j_{s}} \delta(x-y), \text { for } \mathrm{t}+\mathrm{s}>2 \mathrm{n}, \\
& \left\{J_{0}^{i_{t}}(x), J_{0}^{j_{s}}(y)\right\}=\left(J_{0}^{l_{t+s}}-J_{1}^{l_{t+s}}\right)(x) f_{l_{t+s} k_{2 n-s}}^{i_{t}} K^{k_{2 n-s} j_{s}} \delta(x-y), \text { for } \mathrm{t}+\mathrm{s}<2 \mathrm{n} .
\end{aligned}
$$

We would like to stress that the case $n=2$ was previously studied in the context of the pure spinor superstring on $A d S_{5} \times S_{5}$. The algebra of left-invariant currents was also determined in [22. Then it is easy to see that the Poisson brackets (4.14) coincide with the Poisson brackets derived there if we omit the contributions of the ghost fields. This agreement serves as further justification of our result. 


\section{Hamiltonian and equations of motion}

In this section we introduce of the Hamiltonian for the non-linear sigma model on supercoset target. Using the action given in (2.25) we obtain the matter part of the Hamiltonian in the form

$$
H_{\text {matt }}=\int d x \operatorname{Str}\left(\partial_{0} J \Pi-\mathcal{L}\right)=\frac{1}{2} \int d x \operatorname{Str}\left(J_{0} J_{0}+J_{1} J_{1}\right)
$$

or alternatively

$$
\begin{aligned}
H_{\text {matt }} & =\frac{1}{2} \int d x \sum_{r}\left(J_{0}^{i_{r}} K_{i_{r} j_{2 n-r}} J_{0}^{j_{2 n-r}}+J_{1}^{i_{r}} K_{i_{r} j_{2 n-r}} J_{1}^{j_{2 n-r}}\right) \\
& =\frac{1}{2} \int d x \sum_{r}\left((-1)^{|r|} J_{i_{r}}^{0} K^{i_{r} j_{2 n-r}} J_{j_{2 n-r}}^{0}+(-1)^{|r|} J_{i_{r}}^{1} K^{i_{r} j_{2 n-r}} J_{j_{2 n-r}}^{1}\right) .
\end{aligned}
$$

Following the general theory of constraint systems we have to introduce to the Hamiltonian the contribution that corresponds to the fact that the dynamics of the system is restricted on the constraint surface $\Phi_{x}=0$

$$
H_{c o n}=\int d x \Gamma^{x} \Phi_{x}(x)
$$

It can be shown that the time evolution of the primary constraints $\Phi_{x}$ does not induce any secondary constraints so that the whole Hamiltonian takes the form

$$
H=H_{\text {matt }}+H_{\text {con }}
$$

With the help of the Hamiltonian (5.4) we can determine the equations of motion for $J_{i_{r}}^{0}, J_{1}^{i_{s}}$ using the fact that the time evolution of any function $X(J, \Pi)$ that is defined on the phase space spanned by $J_{1}^{i_{A}}, \Pi_{j_{B}}$ is governed by the equation

$$
\partial_{0} X=\{X, H\}
$$

With the help of the Poisson bracket that were derived in the previous section and the form of the Hamiltonian given above we immediately obtain the equation of motion for $J_{1}^{i_{s}}$ in the form

$$
\partial_{0} J_{1}^{i_{r}}=\partial_{x} J_{0}^{i_{r}}+J_{1}^{x} J_{0}^{j_{r}} f_{x j_{r}}^{i_{r}}-\sum_{t} J_{0}^{j_{t}} J_{1}^{l_{r-t}} f_{j_{t} l_{r-t}}^{i_{r}}+\Gamma^{x} J_{1}^{j_{r}} f_{x j_{r}}^{i_{r}} .
$$

The form of the equation above suggests that it is natural to choose the Lagrange multiplier $\Gamma^{x}$ to be equal to (In other words we fix the gauge)

$$
\Gamma^{x}=-J_{0}^{x}
$$


and introduce the covariant derivative

$$
\begin{aligned}
\nabla_{\mu} X_{\nu}^{i_{r}} & \equiv \partial_{\mu} X_{\nu}^{i_{r}}+J_{\mu}^{x} X_{\nu}^{j_{r}} f_{x j_{r}}^{i_{r}} \\
\nabla_{\mu} X_{i_{r}}^{\nu} & \equiv \partial_{\mu} X_{i_{r}}^{\nu}-J^{x} X_{j_{r}}^{\nu} f_{x i_{r}}^{j_{r}} \\
\nabla_{\mu} X_{i_{r}}^{\nu} & =K_{i_{r} j_{2 n-r}} \nabla_{\mu} X_{\nu}^{j_{2 n-r}} .
\end{aligned}
$$

Then we can rewrite the equation (5.6) into the form

$$
-\nabla_{0} J_{1}^{i_{r}}+\nabla_{1} J_{0}^{i_{r}}-\sum_{t} J_{0}^{j_{t}} J_{1}^{l_{r-t}} f_{j_{t} l_{r-t}}^{i_{t}}=0 .
$$

In the same way we can determine the equation of motion for $J_{i_{r}}^{0}$

$$
\begin{aligned}
& -\nabla_{0} J_{i_{r}}^{0}+\nabla_{1} J_{i_{r}}^{1}-\sum_{t}(-1)^{|r||t|+|t|} J_{l_{r+t}}^{0} J_{0}^{j_{t}} f_{i_{r} j_{t}}^{l_{r+t}}- \\
& -\sum_{r+t<2 n}(-1)^{|t|+|r||t|} J_{l_{r+t}}^{1} J_{0}^{j_{t}} f_{i_{r} j_{t}}^{l_{r+t}}+\sum_{r+t>2 n}(-1)^{|t|+|r||t|} J_{l_{r+t}}^{1} J_{0}^{j_{t}} f_{i_{r} j_{t}}^{l_{r+t}}+ \\
& +\sum_{r+t \neq 2 n} J_{1}^{l_{t-r}} f_{i_{r} l_{t-r}}^{j_{t}} K_{j_{t} k_{2 n-t}} J_{1}^{k_{2 n-t}}=0,
\end{aligned}
$$

where we have also used (5.7). Alternatively, using the currents $J_{\mu}^{i_{r}}$ we can rewrite (5.10) into the form

$$
\begin{aligned}
& -\nabla_{0} J_{0}^{i_{r}}+\nabla_{1} J_{1}^{i_{r}}+\sum_{t} J_{0}^{k_{r-t}} J_{0}^{l_{t}} f_{k_{r-t} l_{t}}^{i_{r}}+\sum_{r+t \neq 2 n} J_{1}^{k_{r-t}} J_{1}^{l_{t}} f_{k_{r-t} l_{t}}^{i_{r}}- \\
& -\sum_{t-r<0} J_{1}^{j_{t}} J_{0}^{k_{r-t}} f_{j_{t} k_{r-t}}^{i_{r}}+\sum_{t-r>0} J_{1}^{j_{t}} J_{0}^{k_{r-t}} f_{j_{t} k_{r-t}}^{i_{r}}=0 .
\end{aligned}
$$

The form of the equation (5.11) simplifies for $r=1$ and for $r=2 n-1$. In case $r=1$ it is natural to combine (5.9) with (5.11) and we obtain

$$
\left(\eta^{\mu \nu}-\epsilon^{\mu \nu}\right) \nabla_{\mu} J_{\nu}^{i_{1}}+\left(\eta^{\mu \nu}-\epsilon^{\mu \nu}\right) \sum_{t=2}^{2 n-2} J_{\mu}^{k_{2 n+1-t}} J_{\nu}^{l_{t}} f_{k_{2 n+1-t} l_{t}}^{i_{1}}=0 .
$$

On the other hand for $r=2 n-1$ we subtract (5.9) from (5.11) and we get

$$
\left(\eta^{\mu \nu}+\epsilon^{\mu \nu}\right) \nabla_{\mu} J_{\nu}^{i_{2 n-1}}+\left(\eta^{\mu \nu}+\epsilon^{\mu \nu}\right) \sum_{t=1}^{2 n-2} J_{\mu}^{j_{t}} J_{\nu}^{k_{2 n-1-t}} f_{j_{t} k_{2 n-1-t}}^{i_{2 n-1}}=0
$$

For arbitrary $r$ we can perform the same manipulation. We firstly add (5.9) to (5.11) and we obtain

$$
\begin{array}{r}
\left(\eta^{\mu \nu}-\epsilon^{\mu \nu}\right) \nabla_{\mu} J_{\nu}^{i_{r}}+\eta^{\mu \nu} \sum_{r+t \neq 2 n} J_{\mu}^{k_{r-t}} J_{\nu}^{l_{t}} f_{k_{r-t} l_{t}}^{i_{r}} \\
-\sum_{r+t \neq 2 n} J_{0}^{k_{t}} J_{1}^{l_{r-t}} f_{k_{t} l_{r-t}}^{i_{r}}-\sum_{t-r<0} J_{1}^{k_{t}} J_{0}^{l_{r-t}} f_{k_{t} l_{r-t}}^{i_{r}}+\sum_{t-r>0} J_{1}^{k_{t}} J_{0}^{l_{r-t}} f_{k_{t} l_{r-t}}^{i_{r}}=0 .
\end{array}
$$


On the other hand if we subtract (5.11) from (5.9) we get

$$
\begin{array}{r}
\left(\eta^{\mu \nu}+\epsilon^{\mu \nu}\right) \nabla_{\mu} J_{\nu}^{i_{r}}+\eta^{\mu \nu} \sum_{r+t \neq 2 n} J_{\mu}^{k_{r-t}} J_{\nu}^{l_{t}} f_{k_{r-t} l_{t}}^{i_{r}} \\
+\sum_{r+t \neq 2 n} J_{0}^{k_{t}} J_{1}^{l_{r-t}} f_{k_{t} l_{r-t}}^{i_{r}}-\sum_{t-r<0} J_{1}^{k_{t}} J_{0}^{l_{r-t}} f_{k_{t} l_{r-t}}^{i_{r}}+\sum_{t-r>0} J_{1}^{k_{t}} J_{0}^{l_{r-t}} f_{k_{t} l_{r-t}}^{i_{r}}=0 .
\end{array}
$$

It is easy to see that for $n=2$ these equations of motion coincide with the equations of motion derived in [22] when we again ommit the contributions of ghost fields.

We conclude this section with the derivation of the equation of motion for $J_{1}^{x}$. With the help of the Poisson brackets derived in section (雨) we immediately obtain

$$
\partial_{0} J_{1}^{x}(x)=-\sum_{t}(-1)^{|t|} J_{1}^{j_{2 n-t}} f_{i_{t} j_{2 n-t}}^{x} K^{i_{t} k_{2 n-t}} J_{k_{2 n-t}}^{0}+J_{1}^{w} f_{y w}^{x} \Gamma^{y}-\partial_{x} \Gamma^{x} .
$$

Using (5.7) we can rewrite this equation in the form

$$
\partial_{0} J_{1}^{x}-\partial_{1} J_{0}^{x}+\sum_{t} J_{0}^{i_{t}} J_{1}^{j_{2 n-t}} f_{i_{t} j_{2 n-t}}^{x}+J_{0}^{y} J_{1}^{z} f_{y z}^{x}=0
$$

that is Maurer-Cartan identity for $J_{\mu}^{x}$. In other words the dynamics of $J_{\mu}^{x}$ is trivial.

\section{Global symmetry of the non-linear sigma model}

By definition the left-invariant currents $J^{i_{A}}=\left(g^{-1} d g\right)^{i_{A}}$ (and consequently the action) are invariant under the global symmetry $g^{\prime}=h g$ where $h$ is a constant element from $G$. Our goal is to determine the corresponding conserved charge using standard Noether procedure. To do this let us presume that $h \approx 1+\epsilon$, where $\epsilon(x)=\epsilon^{i_{A}} T_{i_{A}}$ depends on the worldvolume coordinates. Then the variation of the current is equal to

$$
\delta J_{\mu}=g^{-1} \partial_{\mu} \epsilon g
$$

that implies the variations of the components of the currents $J_{\mu}^{i_{A}}$ in the form

$$
\begin{aligned}
\delta J_{\mu}^{i_{A}} & =K^{i_{A} j_{2 n-A}} \operatorname{Str}\left(g T_{j_{2 n-A}} g^{-1} T_{k_{C}}\right) \partial_{\mu} \epsilon^{k_{C}} \\
& =K^{i_{A} j_{2 n-A}} C_{j_{2 n-A} k_{C}} \partial_{\mu} \epsilon^{k_{C}} .
\end{aligned}
$$

It is important to stress that $C_{i_{A} j_{B}}$ is Grassmann odd for $|A+B|=1$ and is Grassmann even for $|A+B|=0$ as follows from the properties of the supertrace and from the fact that the generator $T_{i_{A}}$ is odd matrix for $|A|=1$. 
Now with the help of (6.2) we obtain that the variation of the action is equal to

$$
\delta S=-\int d^{2} x \sum_{t}\left(J_{\mu}^{j_{2 n-t}}\left(\eta^{\mu \nu}-q_{t} \epsilon^{\mu \nu}\right) C_{j_{2 n-t} i_{A}}\right) \partial_{\nu} \epsilon^{i_{A}} \equiv \int d^{2} x \partial_{\nu} \mathcal{J}_{i_{A}}^{\nu} \epsilon^{i_{A}} .
$$

Since for fields that are on-shell any variation of the action has to vanish the expression above implies

$$
\partial_{\mu} \mathcal{J}_{i_{A}}^{\mu}=0
$$

where

$$
\mathcal{J}_{i_{A}}^{\mu}=\sum_{t}\left(\eta^{\mu \nu}-q_{t} \epsilon^{\mu \nu}\right) J_{\nu}^{j_{2 n-t}} C_{j_{2 n-t} i_{A}}
$$

Using (6.4) we can define the conserved charge

$$
q_{i_{A}}=\int d x \mathcal{J}_{i_{A}}^{0}=-\int d x \sum_{t}\left(J_{0}^{j_{2 n-t}}+q_{t} J_{1}^{j_{2 n-t}}\right) C_{j_{2 n-t} i_{A}} .
$$

It is instructive to calculate the Poisson bracket between $q_{i_{A}}$ and $J_{1}^{j_{B}}$. With the help of the Poisson brackets that were determined in section (4) we obtain

$$
\begin{aligned}
& \left\{J_{1}^{i_{t}}(x), q_{j_{B}}\right\}=-K^{i_{t} k_{2 n-t}} \partial_{x} C_{k_{2 n-t} j_{B}}-J_{1}^{l_{t-r}}(x) f_{l_{t-r} m_{r}}^{i_{t}} K^{m_{r} k_{2 n-r}} C_{k_{2 n-r} j_{B}} \\
& \left\{J_{1}^{x}(x), q_{j_{B}}\right\}=-J_{1}^{l_{2 n-r}}(x) f_{l_{2 n-r} m_{r}}^{x} K^{m_{r} k_{2 n-r}} C_{k_{2 n-r} j_{B}} .
\end{aligned}
$$

We can simplify this result using the definition of $C_{i_{A} j_{B}}$ and the properties of leftinvariant currents since

$$
\begin{aligned}
\partial_{x} C_{i_{B} j_{C}} & =\operatorname{Str}\left(\partial_{x} g T_{i_{B}} g^{-1} T_{j_{C}}\right)-\operatorname{Str}\left(T_{i_{B}} q^{-1} \partial_{x} g g^{-1} T_{j_{C}} g\right)= \\
& =\operatorname{Str}\left(g^{-1} \partial_{x} g T_{i_{B}} g^{-1} T_{j_{C}} g\right)-(-1)^{|B||C|} \operatorname{Str}\left(g^{-1} \partial_{x} g g^{-1} T_{j_{C}} g T_{i_{B}}\right)= \\
& =\sum_{A} J^{k_{A}} \operatorname{Str}\left(\left[T_{k_{A}} T_{i_{B}}-(-1)^{|A||B|} T_{i_{B}} T_{k_{A}}\right] g^{-1} T_{j_{C}} g\right)= \\
& =\sum_{A} J^{k_{A}} f_{k_{A} i_{B}}^{l_{D}} \operatorname{Str}\left(T_{l_{D}} g^{-1} T_{j_{C}} g\right)=\sum_{A} J^{k_{A}} f_{k_{A} i_{B}}^{l_{D}} C_{l_{D} j_{C}} .
\end{aligned}
$$

With the help of this result the first equation in (6.7) takes the form

$$
\begin{aligned}
\left\{J_{1}^{i_{t}}(x), q_{j_{B}}\right\} & =\sum_{A}(-1)^{|t||t+A|} J_{1}^{l_{t-A}} f_{l_{t-A} m_{A}}^{i_{t}} K^{m_{A} k_{2 n-A}} C_{k_{2 n-A} j_{B}}(x)- \\
& -\sum_{r} J_{1}^{l_{t-r}} f_{l_{t-r} m_{r}}^{i_{t}} K^{m_{r} k_{2 n-r}} C_{k_{2 n-r} j_{B}}(x) .
\end{aligned}
$$

For $|t|=0$ the equation above simplifies as

$$
\left\{J_{1}^{i_{t}}(x), q_{j_{B}}\right\}=J_{1}^{l_{t}} f_{l_{t} x}^{i_{t}} K^{x y} C_{y j_{B}}=\delta_{\text {gauge }} J_{1}^{i_{t}}(x)\left(C_{y j_{B}}(x)\right) .
$$


In other words the Poisson bracket between $J_{1}^{i_{t}},|t|=0$ and $q_{j_{B}}$ is equal to the gauge transformation of current $J_{1}^{i_{t}}$ with the gauge parameter equal to $C_{y j_{B}}(x)$. However for $|t|=1$ we do not obtain such a clear interpretation since

$$
\begin{aligned}
\left\{J_{1}^{i_{2 s+1}}(x), q_{j_{B}}\right\} & =-2 \sum_{t=1}^{n-1} J_{1}^{l_{2 s+1-2 t}} f_{l_{2 s+1-2 t} m_{2 t}}^{i_{2 s+1}} K^{m_{2 t} k_{2 n-2 t}} C_{k_{2 n-2 t} j_{B}}(x)+ \\
& +J_{1}^{l_{2 s+1}} f_{l_{2 s+1} x}^{i_{2 s+1}} K^{x y} C_{y j_{B}}(x) .
\end{aligned}
$$

We again see that the expression on the second line in (6.11) can be written as $\delta_{\text {gauge }} J^{i_{2 s+1}}\left(C_{y j_{B}}\right)$. On the other hand it is not clear to us how to interpret the expression on the first line.

Finally, using (6.8) the second equation in (6.7) can be written as

$$
\left\{J_{1}^{x}(x), q_{j_{B}}\right\}=\sum_{r} J_{1}^{l_{2 n-r}}(x) f_{l_{2 n-r} x}^{k_{2 n-r}} K^{x y} C_{y j_{B}}=\sum_{r} \delta_{g a u g e} J_{1}^{k_{2 n-r}}\left(C_{y j_{B}}(x)\right)
$$

that can be again interpreted as a sum of the gauge transformations of currents $J_{1}^{i_{t}}$. At present it is not completely clear to us how to interpret these results. Since the left-invariant currents are invariant under the transformations $g^{\prime}=h g$ one could expect that the Poisson brackets between left-invariant currents and the charges corresponding to the global symmetry of the action are either zero or equal to the gauge transformations that define coset. We have seen that this interpretation holds for Grassmann even components of the left-invariant currents. On the other hand we have derived different results for the Grassmann odd components of the left-invariant currents and as we mentioned above it is not clear to us how to interpret this result. This issue certainly deserve more precise study and we hope to return to it in future.

\section{Acknowledgements}

We would like to thank M. Bianchi and A. Das for useful discussions. This work was supported in part by INFN, by the MIUR-COFIN contract 2003-023852, by the EU contracts MRTN-CT-2004-503369 and MRTN-CT-2004-512194, by the INTAS contract 03-516346 and by the NATO grant PST.CLG.978785 and in part by the Czech Ministry of Education under Contract No. MSM 0021622409.

\section{References}

[1] S. Guruswamy, A. LeClair and A. W. W. Ludwig, "Gl(N|N) Super-Current Algebras For Disordered Dirac Fermions In Two Dimensions," Nucl. Phys. B 583 (2000) 475 [arXiv:cond-mat/9909143].

[2] N. Read and H. Saleur, "Exact spectra of conformal supersymmetric nonlinear sigma models in two dimensions," Nucl. Phys. B 613 (2001) 409 [arXiv:hep-th/0106124]. 
[3] P. A. Grassi and M. Marescotti, "Flux vacua and supermanifolds," arXiv:hepth/0607243.

[4] R. R. Metsaev and A. A. Tseytlin, "Type IIB superstring action in AdS(5) $x$ S(5) background," Nucl. Phys. B 533 (1998) 109 [arXiv:hep-th/9805028].

[5] R. Roiban and W. Siegel, "Superstrings on AdS(5) x S(5) supertwistor space," JHEP 0011 (2000) 024 [arXiv:hep-th/0010104].

[6] M. Hatsuda and M. Sakaguchi, "Wess-Zumino term for AdS superstring," Phys. Rev. D 66 (2002) 045020 [arXiv:hep-th/0205092].

[7] M. Hatsuda and K. Kamimura, "Classical AdS superstring mechanics," Nucl. Phys. B 611 (2001) 77 [arXiv:hep-th/0106202].

[8] M. Bershadsky, S. Zhukov and A. Vaintrob, "PSL $(n \mid n)$ sigma model as a conformal field theory," Nucl. Phys. B 559 (1999) 205 [arXiv:hep-th/9902180].

[9] N. Berkovits, C. Vafa and E. Witten, "Conformal field theory of AdS background with Ramond-Ramond flux," JHEP 9903 (1999) 018 [arXiv:hep-th/9902098].

[10] N. Berkovits, M. Bershadsky, T. Hauer, S. Zhukov and B. Zwiebach, "Superstring theory on $A d S(2) x S(2)$ as a coset supermanifold," Nucl. Phys. B 567 (2000) 61 [arXiv:hep-th/9907200].

[11] I. Bena, J. Polchinski and R. Roiban, "Hidden symmetries of the AdS(5) $x S^{* *} 5$ superstring," Phys. Rev. D 69 (2004) 046002 [arXiv:hep-th/0305116].

[12] B. Chen, Y. L. He, P. Zhang and X. C. Song, "Flat currents of the Green-Schwarz superstrings in $\operatorname{AdS(5)} x S^{* * 1}$ and $A d S(3) x S^{* * 3}$ backgrounds," Phys. Rev. D 71, 086007 (2005) [arXiv:hep-th/0503089].

[13] B. C. Vallilo, "Flat currents in the classical $A d S(5) x S^{* * 5}$ pure spinor superstring," JHEP 0403 (2004) 037 [arXiv:hep-th/0307018].

[14] N. Berkovits, "BRST cohomology and nonlocal conserved charges," JHEP 0502 (2005) 060 [arXiv:hep-th/0409159].

[15] N. Berkovits, "Quantum consistency of the superstring in $A d S(5) x S^{* *} 5$ background," JHEP 0503 (2005) 041 [arXiv:hep-th/0411170].

[16] B. C. Vallilo, "One loop conformal invariance of the superstring in an $A d S(5) x S(5)$ background," JHEP 0212 (2002) 042 [arXiv:hep-th/0210064].

[17] D. Kagan and C. A. S. Young, "Conformal Sigma-Models On Supercoset Targets," Nucl. Phys. B 745 (2006) 109 [arXiv:hep-th/0512250].

[18] C. A. S. Young, "Non-Local Charges, Z(M) Gradings And Coset Space Actions," Phys. Lett. B 632 (2006) 559 [arXiv:hep-th/0503008]. 
[19] L. D. Faddeev and L. A. Takhtajan, "HAMILTONIAN METHODS IN THE THEORY OF SOLITONS,"

[20] A. Das, J. Maharana, A. Melikyan and M. Sato, "The algebra of transition matrices for the AdS(5) x $S^{* * 5}$ superstring," JHEP 0412, 055 (2004) [arXiv:hep-th/0411200].

[21] A. Das, A. Melikyan and M. Sato, "The algebra of flat currents for the string on AdS(5) $x S^{* * 5}$ in the light-cone gauge," JHEP 0511, 015 (2005) [arXiv:hep-th/0508183].

[22] M. Bianchi and J. Kluson, "Current Algebra Of The Pure Spinor Superstring In Ads(5) $X S(5)$, , arXiv:hep-th/0606188.

[23] D. Korotkin and H. Samtleben, "Yangian symmetry in integrable quantum gravity," Nucl. Phys. B 527 (1998) 657 [arXiv:hep-th/9710210].

[24] B. H. Miller, "Conserved charges in the principal chiral model on a supergroup," JHEP 0608 (2006) 010 [arXiv:hep-th/0602006]. 